\title{
Modified FOLFOX-6 Plus Bevacizumab Chemotherapy for Metastatic Colorectal Cancer in Patients Receiving Hemodialysis: A Report of Three Cases and Review of the Literature
}

\author{
Chikako Funasaka $^{a} \quad$ Yusuke Kanemasa $^{a} \quad$ Tatsu Shimoyama $^{a}$ \\ Akihito Ohtab $^{\mathrm{b}}$ Yasushi Omuro ${ }^{\mathrm{a}}$ \\ aDepartment of Medical Oncology, Tokyo Metropolitan Cancer and Infectious Diseases \\ Center Komagome Hospital, Tokyo, Japan; bepartment of Nephrology, Tokyo \\ Metropolitan Cancer and Infectious Diseases Center Komagome Hospital, Tokyo, Japan
}

\section{Keywords}

Colorectal cancer $\cdot$ Hemodialysis $\cdot$ Chemotherapy

\begin{abstract}
Fluorouracil plus oxaliplatin (L-OHP) (FOLFOX) plus bevacizumab (BV) therapy is commonly administered to patients with metastatic colorectal cancer. However, few reports have described L-OHP therapy in hemodialysis patients, and the efficacy and safety remain uncertain in this population. Here, we report three cases of hemodialysis patients with colorectal cancer who received a modified FOLFOX-6 (mFOLFOX-6, or FOLFOX plus folinic acid) plus BV regimen every 3 weeks. One patient, a 65 -year-old man with chronic renal failure consequent to diabetic nephropathy, underwent hemodialysis 3 times/week. He exhibited a partial response after 7 cycles of mFOLFOX-6 plus BV, with the major adverse events of Grade 1 peripheral neuropathy and Grade 2 thrombocytopenia. He died of perforation-related septic shock. A 71-year-old
\end{abstract}




\section{Case Reports in Oncology}

Case Rep Oncol 2019;12:657-665

DOI: $10.1159 / 000502512$

(c) 2019 The Author(s). Published by S. Karger AG, Base www.karger.com/cro

Funasaka et al.: Modified FOLFOX-6 Plus Bevacizumab Chemotherapy for Metastatic Colorectal Cancer in Patients Receiving Hemodialysis

man previously treated with bosutinib for chronic myelocytic leukemia received 9 cycles of mFOLFOX-6 plus BV and achieved stable disease. Chemotherapy was administered every 4 weeks, and the 5-fluorouracil dose was reduced after he developed Grade 4 neutropenia. A 71-year-old woman with chronic renal failure consequent to diabetic nephropathy underwent hemodialysis 3 times a week. She received 3 cycles of mFOLFOX-6 plus BV, but exhibited disease progression and developed Grade 4 neutropenia, which necessitated a reduced 5 -fluorouracil dose. After completing FOLFOX therapy, she began second-line irinotecan/5-fluoroura$\mathrm{cil} /$ leucovorin (FOLFIRI) plus BV therapy. In two cases, bone marrow suppression increased the difficulty of L-OHP dose escalation. We conclude that mFOLFOX-6 plus BV, with appropriate dose reduction, is acceptable for patients with chronic renal failure. Further data are needed to determine the adequate chemotherapy dose.

(C) 2019 The Author(s)

Published by S. Karger AG, Basel

\section{Introduction}

The global incidence of colorectal cancer, the third leading cause of cancer-related death, continues to increase [1]. Modified FOLFOX-6 (mFOLFOX-6; oxaliplatin (L-OHP) with 5-fluorouracil (5-FU) and folinic acid) plus bevacizumab (BV) is a standard chemotherapy regimen for metastatic colorectal cancer. This treatment was reported to yield an objective response rate of $52 \%$ and median time to treatment failure of 5.8 months [2].

Although long-term hemodialysis is a significant risk factor for cancer [3], only a few reports have described the use of FOLFOX therapy in patients undergoing chronic hemodialysis. Therefore, the efficacy and safety of FOLFOX therapy in this population remains unclear. Herein, we report 3 cases involving patients undergoing chronic hemodialysis who received mFOLFOX-6 plus BV for metastatic colorectal cancer. All three received mFOLFOX-6 as described by Horimatsu et al. [4], with hemodialysis initiation immediately after L-OHP infusion. Treatment efficacy was evaluated according to the Response Evaluation Criteria in Solid Tumors (RECIST) 1.1 criteria, and adverse events were evaluated according to the Common Terminology Criteria for Adverse Events (CTCAE) v 4.0.

\section{Case Report/Case Presentation}

\section{Case 1}

A 65-year-old man with chronic renal failure consequent to diabetic nephropathy had undergone hemodialysis 3 times/week at another hospital since March 2017. Screening prior to hemodialysis induction revealed a moderately/poorly differentiated adenocarcinoma in the ascending colon. The tumor was G13C KRAS mutation-positive but BRAF mutation-negative. Computed tomography (CT) revealed a $20-\mathrm{mm}$ metastasis to S6/7 of the liver. The patient was diagnosed with stage IV colon cancer and underwent a right colectomy in April 2017, followed by resection of the liver metastasis in July 2017. Postoperative CT revealed multiple lung metastases, liver metastasis, lymph node metastasis, and ascites.

The patient began receiving chemotherapy at our hospital in November 2017. At that time, his ECOG performance status, body surface area (BSA), and body weight were $1,1.88 \mathrm{~m}^{2}$, 
and $76.9 \mathrm{~kg}$, respectively. He began receiving mFOLFOX-6 (L-OHP: $60 \mathrm{mg} / \mathrm{m}^{2}$ [112 mg], l-LV: $200 \mathrm{mg} / \mathrm{m}^{2}$ [375 mg], bolus 5-FU: $400 \mathrm{mg} / \mathrm{m}^{2}$ [751 mg], 46-hr injection of 5-FU: 2,400 mg/m² [4,500 mg]) plus BV (5 mg/kg [384 mg]) in December 2017. He received 7 cycles of this therapy over a period of 4.7 months, with L-OHP dose escalation in the fifth cycle. His serum platinum concentration over time is shown in Figure 1. This patient achieved a partial response and developed the major adverse events of Grade 1 peripheral neuropathy and Grade 2 thrombocytopenia. He died of perforation-related septic shock at the other hospital.

Case 2

A 71-year-old man with chronic renal failure consequent to diabetic nephropathy had undergone hemodialysis 3 times/week since February 2017. He had a history of chronic myelocytic leukemia for which he had received bosutinib in the hematology department of our hospital. An examination revealed anemia, and a colonoscopy identified a KRAS and BRAF mutation-negative type 2 adenocarcinoma (tub1/ tub2) in the rectum. He underwent laparoscopic colectomy and partial resection of the intestine, which led to a diagnosis of stage IIIa rectal adenocarcinoma. No adjuvant chemotherapy was administered.

In April 2018, CT revealed multiple liver metastases and evidence of local recurrence. At that time, his performance status, BSA, and body weight were $0,1.7 \mathrm{~m}^{2}$, and $55.9 \mathrm{~kg}$, respectively. He began receiving mFOLFOX-6 (L-OHP: $60 \mathrm{mg} / \mathrm{m}^{2}$ [100 mg], l-LV: $200 \mathrm{mg} / \mathrm{m}^{2}$ [334 $\mathrm{mg}$ ], bolus 5-FU: $400 \mathrm{mg} / \mathrm{m}^{2}$ [669 mg], 46-h injection of 5-FU: 2,400 mg/m² [4,015 mg]) plus BV (5 mg/kg [279 mg]) in May 2018 and received a total of 9 chemotherapy cycles in 7.7 months. He achieved stable disease but developed Grade 4 bone marrow suppression, necessitating a 5-FU dose reduction (bolus 5-FU: $320 \mathrm{mg} / \mathrm{m}^{2}$ [534 mg], 46-h injection of 5-FU: 1,920 $\left.\mathrm{mg} / \mathrm{m}^{2}[3,200 \mathrm{mg}]\right)$ from the second treatment course and a decrease in the chemotherapy frequency to every 4 weeks. His serum platinum concentration over time is shown in Figure 2. The patient developed the major adverse events of Grade 4 neutropenia and Grade 3 leukopenia. No non-hematological adverse events were observed. He continues to receive treatment.

Case 3

A 71-year-old woman with chronic renal failure consequent to diabetic nephropathy had undergone hemodialysis 3 times/week since August 2017. She presented with anemia, and a colonoscopy revealed a G12D KRAS mutation-positive, $B R A F$ mutation-negative type 2 adenocarcinoma (tub2 > por2) in the transverse colon. As CT revealed a liver metastasis, the patient was diagnosed with Stage IV colorectal cancer and underwent a laparoscopic right colectomy in September 2017, followed by a resection of the liver metastasis in December 2017.

In May 2018, a postoperative CT examination revealed multiple lung metastases and a recurrence of the liver metastasis. At that time, her performance status, BSA, and body weight were $2,1.4 \mathrm{~m}^{2}$, and $52.8 \mathrm{~kg}$, respectively. She began receiving mFOLFOX-6 (L-OHP: $60 \mathrm{mg} / \mathrm{m}^{2}$ [86 mg], l-LV: $200 \mathrm{mg} / \mathrm{m}^{2}$ [288 mg], bolus 5-FU: $400 \mathrm{mg} / \mathrm{m}^{2}$ [576 mg], 46-h injection of 5-FU: $2,400 \mathrm{mg} / \mathrm{m}^{2}$ [3,400 mg]) plus BV (5 mg/kg [264 mg]). However, she developed Grade 4 bone marrow suppression which necessitated a 5-FU dose reduction (bolus 5 -FU: $320 \mathrm{mg} / \mathrm{m}^{2}$ [460 $\mathrm{mg}$ ], $46-\mathrm{h}$ injection of $5-\mathrm{FU}: 1,920 \mathrm{mg} / \mathrm{m}^{2}$ [2,720 mg]) from the second chemotherapy course. She received 3 cycles of mFOLFOX-6 therapy over a period of 2.2 months but exhibited progressive disease with major adverse events of Grade 4 neutropenia, Grade 3 thrombocyto- 
penia, and Grade 1 fatigue. Her serum platinum concentration over time is shown in Figure 3. FOLFOX therapy was discontinued, and irinotecan/5-fluorouracil/leucovorin (FOLFIRI) plus BV therapy was initiated as a second-line treatment.

\section{Discussion/Conclusion}

In this case series, we have demonstrated the acceptability of mFOLFOX-6 plus BV for colorectal cancer in patients undergoing chronic hemodialysis. However, two patients developed severe hematological adverse events such as bone marrow suppression, which presented challenges with respect to L-OHP dose escalation.

Table 1 lists the published cases involving FOLFOX therapy for colorectal cancer in patients receiving chronic hemodialysis [4, 5-13]. In all listed cases, the L-OHP dose was reduced to $50-80 \%$ of the standard dose $\left(85 \mathrm{mg} / \mathrm{m}^{2}\right)$ and in most, hemodialysis was initiated immediately after L-OHP infusion. In all previously reported cases, FOLFOX therapy was administered safely without any severe adverse events and although the efficacy of this treatment is unclear, tumor progression was not observed in most cases. In our cases, the starting L-OHP dose was $60 \mathrm{mg} / \mathrm{m}^{2}$, with the intent to escalate to $85 \mathrm{mg} / \mathrm{m}^{2}$. However, this was difficult due to myelosuppression. Major hematological adverse events, such as thrombocytopenia and neutropenia, were observed. Although Grade 4 neutropenia occurred in two patients aged $>70$ years, febrile neutropenia was not observed. These adverse events were managed adequately by appropriate dose reductions and increased intervals between subsequent chemotherapy cycles. No severe non-hematological toxicities (e.g., peripheral neuropathy) were observed in our three patients.

The pharmacokinetic data in previous reports demonstrate that free platinum levels follow a bimodal pattern, with peaks appearing at 2 and $26 \mathrm{~h}$ after the start of L-OHP administration. The second peak appears after the first hemodialysis session and is attributed to the dissociation of platinum bound to plasma proteins and blood cells or to the return of platinum from the tissues to the blood. In patients receiving hemodialysis, this second peak has a greater influence on the area under the curve than that observed with FOLFOX therapy in patients with normal renal function. Therefore, patients receiving hemodialysis generally are generally treated at intervals of 3 weeks, which is longer than the interval used for FOLFOX therapy in patients with normal renal function.

A previous study by Giacchetti et al. reported differences in hematological toxicity between patients treated with FOLFOX-2 and a chronomodulated infusion of L-OHP, 5-FU, and leucovorin (chronoFLO4) and suggested that hematological toxicity correlates with the $\mathrm{C}_{\max }$ of L-OHP [13]. In our three patients, the $\mathrm{C}_{\max }$ for a L-OHP dose of $60 \mathrm{mg} / \mathrm{m}^{2}$ was approximately $50-94 \%$ of that observed with for a L-OHP dose of $90 \mathrm{mg} / \mathrm{m}^{2}$ in patients with normal renal function [14]. Although the $\mathrm{C}_{\max }$ values of serum platinum concentrations in our cases were similar to those in other reports, our patients exhibited more severe hematological toxicities.

Moreover, the plasma platinum concentrations before L-OHP administration and the platinum $\mathrm{C}_{\max }$ increased gradually in our patients with each treatment course, and hematological adverse events occurred from the first therapy cycle. In other words, we did not observe a relationship between $\mathrm{C}_{\max }$ and the severity of hematological toxicity, possibly because we administered appropriate doses of 5-FU. Our findings may be consistent with other reports that 
did not suggest a relationship between the $\mathrm{C}_{\max }$ of L-OHP and hematological toxicity. An understanding of the correlation between toxicity and L-OHP pharmacokinetics in hemodialysis patients should also consider concentrations of other drugs in the combination regimen.

In conclusion, our cases demonstrate the acceptability of the mFOLFOX-6 plus BV regimen for the treatment of advanced colorectal cancer in patients receiving hemodialysis. However, it is necessary to monitor toxicity carefully in these patients, given the tendency of adverse events to be severe and the consequent need for L-OHP dose reduction. Further investigations should aim to clarify the dose and chemotherapy intervals to allow adjustments according to plasma platinum pharmacokinetics and the occurrence of adverse events.

\section{Statement of Ethics}

We have reported these cases in compliance with the Declaration of Helsinki. General consent for the publication and presentation of case data were obtained from the patients when they provided informed consent to undergo treatment.

\section{Disclosure Statement}

The authors have no conflicts of interest to declare.

\section{Funding Sources}

No funding was received for this research.

\section{Author Contributions}

We thank the patients and Dr. Kentaro Kawasoe and Dr. Teruhiro Fujii, the treating nephrologists, for their cooperation with this study.

\section{References}

1 Bray F, Ferlay J, Soerjomataram I, Siegel RL, Torre LA, Jemal A. Global cancer statistics 2018: GLOBOCAN estimates of incidence and mortality worldwide for 36 cancers in 185 countries. CA Cancer J Clin. 2018 Nov;68(6):394-424.

2 Hochster HS, Hart LL, Ramanathan RK, Childs BH, Hainsworth JD, Cohn AL, et al. Safety and efficacy of oxaliplatin and fluoropyrimidine regimens with or without bevacizumab as first-line treatment of metastatic colorectal cancer: results of the TREE Study. J Clin Oncol. 2008 Jul;26(21):3523-9.

3 Maisonneuve P, Agodoa L, Gellert R, Stewart JH, Buccianti G, Lowenfels AB, et al. Cancer in patients on dialysis for end-stage renal disease: an international collaborative study. Lancet. 1999 Jul;354(9173):93-9.

4 Horimatsu T, Miyamoto S, Morita S, Mashimo Y, Ezoe Y, Muto M, et al. Pharmacokinetics of oxaliplatin in a hemodialytic patient treated with modified FOLFOX- 6 plus bevacizumab therapy. Cancer Chemother Pharmacol. 2011 Jul;68(1):263-6. 
5 Ohnishi T, Kanoh T, Shiozaki K, Kimura Y, Iwazawa T, Tono T, et al. [FOLFOX 4 in a patient with metastatic colorectal cancer on hemodialysis due to chronic renal failure]. Gan To Kagaku Ryoho. 2007 Aug;34(8):1299-302. Japanese.

6 Matoba S, Sawada T, Toda S, Moriyama J, Yokoyama T, Ehara K, et al. [Modified FOLFOX6 in a patient on hemodialysis with metastatic colorectal cancer]. Gan To Kagaku Ryoho. 2008 Apr;35(4):673-5. Japanese.

7 Katsumata K, Sumi T, Wada T, Mori Y, Hisada M, Kawakita H, et al. Oxaliplatin for metastatic colon cancer in a patient with renal failure. Clin Med Oncol. 2008;2:97-101.

8 Fujita M, Koide T, Katayama T, Matsuda H, Yamagishi Y, Okuda M, et al. [The pharmacokinetics and safety of oxaliplatin in a hemodialysis patient treated with mFOLFOX6 therapy]. Gan To Kagaku Ryoho. 2009 Aug;36(8):1379-82.

9 Watayo Y, Kuramochi H, Hayashi K, Nakajima G, Kamikozuru H, Yamamoto M. Drug monitoring during FOLFOX6 therapy in a rectal cancer patient on chronic hemodialysis. Jpn J Clin Oncol. 2010 Apr;40(4):360-4

10 Kuwabara H, Baba H, Wakabayashi M, Nakamura H, Sanada T, Baba H, et al. [mFOLFOX6 and FOLFIRI/bevacizumab treatment in a patient on hemodialysis with metastatic colon cancer]. Gan To Kagaku Ryoho. 2011 Nov;38(12):2250-2.

11 Gori S, Lunardi G, Inno A, Magarotto R, Duranti S, Messa MG, et al. Pharmacokinetics of oxaliplatin in a hemodialyzed patient: chemotherapy dose adjustment and timing of dialysis. Clin Colorectal Cancer. 2014 Dec;13(4):260-3.

12 van Berlo-van de Laar IR, Brummelhuis WJ, Imholz AL, Schellens JH, Huitema AD, Jansman FG. Dosing oxaliplatin in a haemodialysis patient with metastatic rectum cancer monitored by free platinum concentrations. J Clin Pharm Ther. 2018 Aug;43(4):574-7.

13 Giacchetti S, Bjarnason G, Garufi C, Genet D, Iacobelli S, Tampellini M, et al.; European Organisation for Research and Treatment of Cancer Chronotherapy Group. Phase III trial comparing 4-day chronomodulated therapy versus 2-day conventional delivery of fluorouracil, leucovorin, and oxaliplatin as first-line chemotherapy of metastatic colorectal cancer: the European Organisation for Research and Treatment of Cancer Chronotherapy Group. J Clin Oncol. 2006 Aug;24(22):3562-9.

14 Shirao K, Matsumura Y, Yamada Y, Muro K, Gotoh M, Boku N, et al. Phase I study of single-dose oxaliplatin in Japanese patients with malignant tumors. Jpn J Clin Oncol. 2006 May;36(5):295-300. 


\section{Case Reports in Oncology}

Case Rep Oncol 2019;12:657-665

DOI: $10.1159 / 000502512$

1

(C) 2019 The Author(s). Published by S. Karger AG, Basel www.karger.com/cro

Funasaka et al:: Modified FOLFOX-6 Plus Bevacizumab Chemotherapy for Metastatic Colorectal Cancer in Patients Receiving Hemodialysis

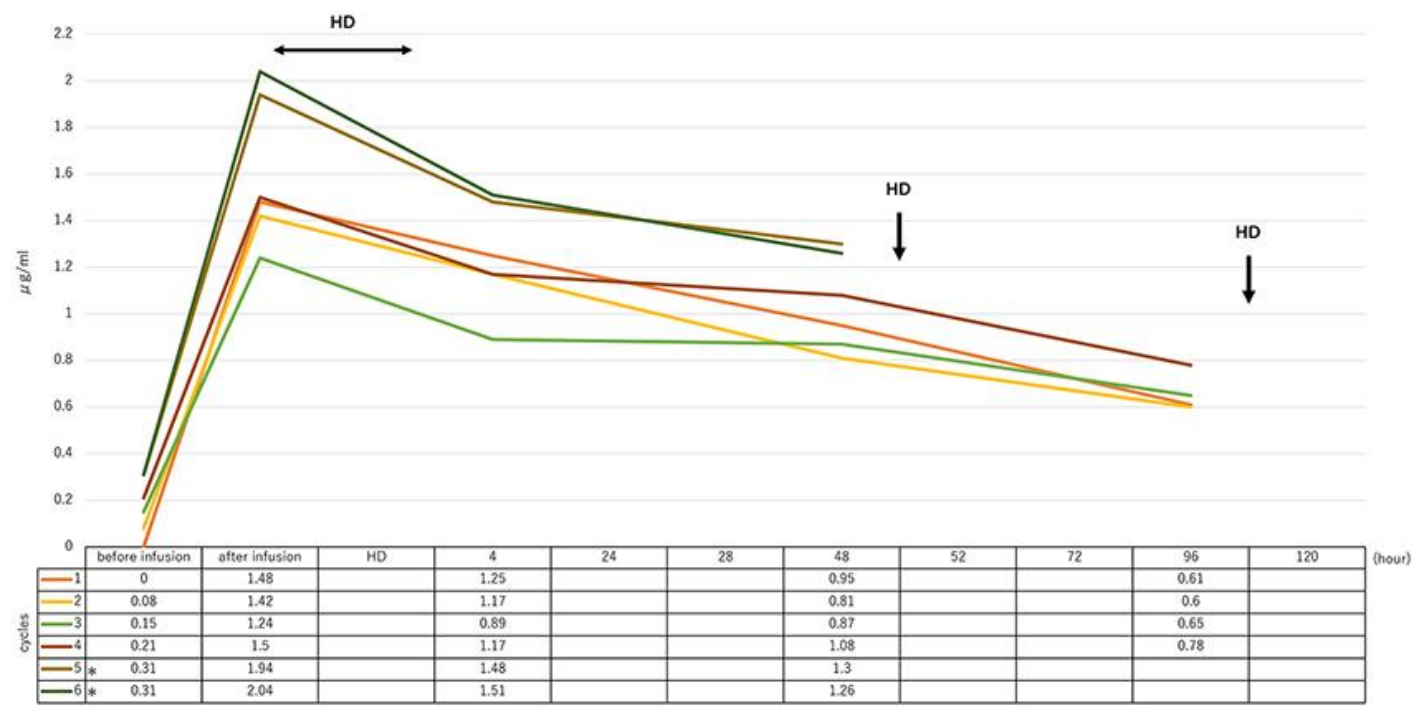

Fig. 1. Serum platinum concentrations over time in Case 1. * Oxaliplatin dose of $70 \mathrm{mg} / \mathrm{m} 2$. HD, hemodialysis.

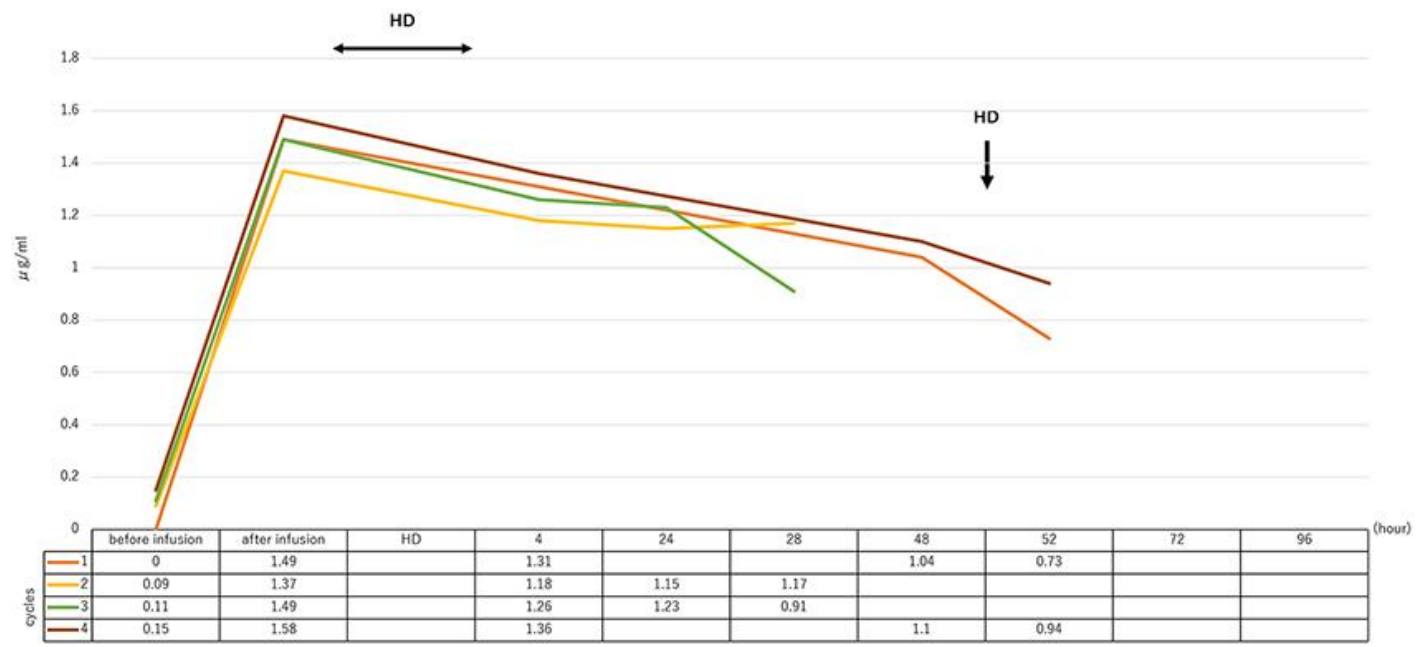

Fig. 2. Serum platinum concentrations over time in Case 2. In cycles 2 and 3, temporary hemodialysis was performed $24 \mathrm{~h}$ after oxaliplatin infusion because of insufficient drainage. HD, hemodialysis. 


\section{Case Reports in Oncology}

Funasaka et al.: Modified FOLFOX-6 Plus Bevacizumab Chemotherapy for Metastatic Colorectal Cancer in Patients Receiving Hemodialysis

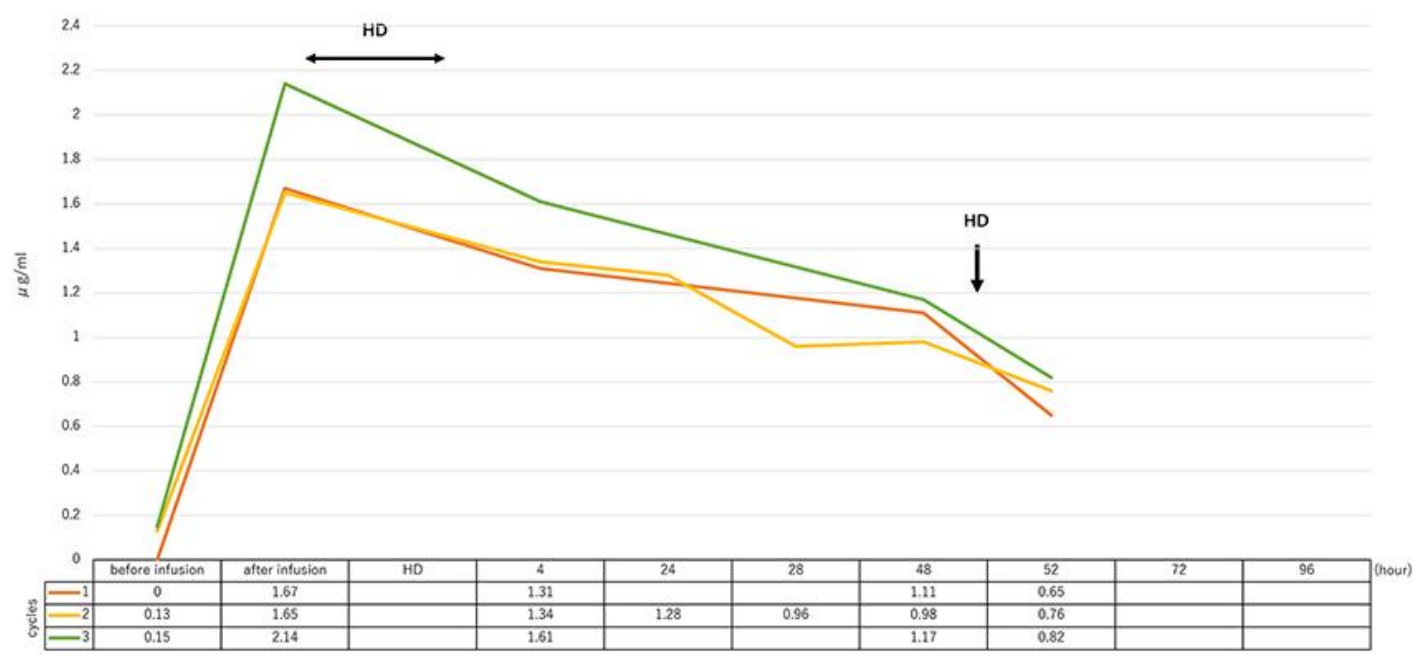

Fig. 3. Serum platinum concentrations over time in Case 3. In cycle 2, temporary hemodialysis was performed $24 \mathrm{~h}$ after oxaliplatin infusion because of insufficient drainage. HD, hemodialysis. 
Table 1. Summary of data from previous case reports

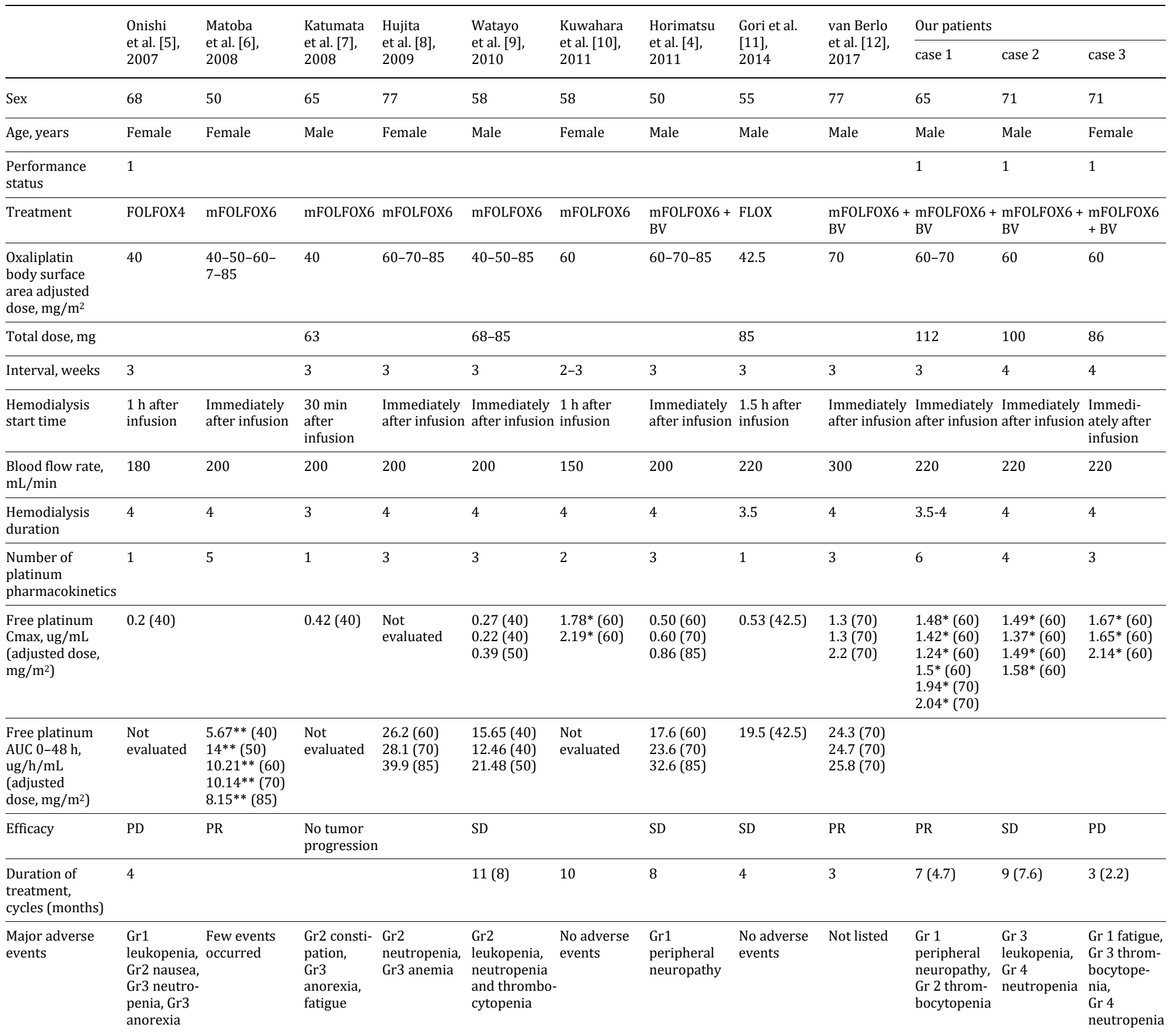

* Plasma total platinum Cmax, ** AUC: 0-26 h. Gr, Grade; AUC, area under the curve; PR, partial response; SD, stable disease; PD, progressive disease. 